\title{
Nucleon Electromagnetic Form Factors and Quark Content
}

\section{Gordon Cates*广}

University of Virginia, Charlottesville

E-mail: cates@virginia.edu

\begin{abstract}
A brief summary of the status of measurements of the ground-state electromagnetic nucleon form factors is presented, along with a discussion of the implications for nucleon structure. The discovery that the ratio of the electric and magnetic form factors of the proton, $G_{E}^{p} / G_{M}^{p}$, decreases almost linearly with increasing $Q^{2}$ has brought considerable new attention to the measurement of nucleon form factors at high $Q^{2}$. A consensus is growing in which the explanation for this behavior can be found in the role played by quark orbital angular momentum. Interestingly, several subsequent experiments have seen evidence for quark OAM in completely independent contexts. Recent measurements of the ratio of the electric and magnetic form factors of the neutron, $G_{E}^{n} / G_{M}^{n}$ have been made up to $Q^{2}=3.4 \mathrm{GeV}^{2}$. It is now possible to disentangle the $u$ - and $d$-quark contributions to the elastic form factors in the $Q^{2}$ regime in which the interesting behavior in the proton was observed. The behavior of the $u$ - and $d$-quark contributions was found to be markedly different, a fact that has been interpreted as evidence for diquark-like structures within the nucleon. We also discuss progress and future plans on precise measurements of the charge radius of the proton, a subject that has gotten considerable attention since the charge radius extracted from a measurement of the Lamb Shift in muonic hydrogen was seen to be in disagreement with charge radii measured in electron scattering. Elastic form factors are having a profound influence over the modern understanding of the QCD structure of the nucleon.
\end{abstract}

Sixth International Conference on Quarks and Nuclear Physics

April 16-20, 2012

Ecole Polytechnique, Palaiseau, Paris

* Speaker.

${ }^{\dagger}$ This work was supported in part by the US Department of Energy under Contract No. DE-FG02-01ER41168 


\section{Introduction}

Measurements of the nucleon elastic form factors provide a critical means for accessing the transverse structure of the nucleon. They provide information on the distribution of charge and magnetization, and some of the strongest constraints on Generalized Parton Distributions (GPDs). Furthermore, by combining data from both the proton and the neutron, and assuming charge symmetry, the nucleon form factors tell us about the distribution of the individual quark flavors. The picture of the nucleon that has emerged from form factor measurements during the last decade has qualitatively changed the picture that existed previously, something that underscores the importance of recent measurements.

A strong reminder of the importance of the nucleon form factors in understanding nucleon structure came when it was discovered at JLab that, for values of the four-momentum transfer $Q^{2}$ above roughly $1 \mathrm{GeV}^{2}$, the ratio of the electric and magnetic form factors of the proton, $G_{E}^{p} / G_{M}^{p}$, decreases with increasing $Q^{2}$ in an almost linear fashion [1]. The observation was made using a double-polarization technique, and has subsequently been confirmed up to $8.5 \mathrm{GeV}^{2}$ [2]. The striking $Q^{2}$ behavior of $G_{E}^{p} / G_{M}^{p}$ has ignited considerable reexamination of nucleon structure, and resulted in a recognition of the importance of the effects of quark orbital angular momentum, as well as the importance of two-photon effects when using traditional Rosenbluth separations.

Recent measurements of the ratio of the electric and magnetic form factors of the neutron, $G_{E}^{n} / G_{M}^{n}$, have been made up to $Q^{2}=3.4 \mathrm{GeV}^{2}$. When taken together with the proton results, it is now possible to extract the individual $u$ - and $d$-quark contributions to the elastic nucleon form factors in the $Q^{2}$-regime in which the interesting behavior of the proton was observed. The flavordecomposed form factors exhibit several surprising behaviors, and seem to suggest there is evidence for diquark-like structures within the nucleon.

At low $Q^{2}$, precise new data from Mainz and JLab provide significantly improved knowledge of the charge radius of the proton. Interestingly, while the Mainz and JLab data agree with one another, as well as with charge-radius determinations extracted from Lamb Shift measurements on hydrogen, they disagree significantly with an accurate new charge-radius measurement extracted from the Lamb Shift in muonic hydrogen.

In the coming years, we can expect a dramatic improvement in our knowledge of the elastic nucleon form factors. The JLab $12 \mathrm{GeV}$ upgrade will roughly double the $Q^{2}$ range over which they will be accessible, probing a regime in which new theoretical decriptions of nucleon structure will be definitively tested. At low $Q^{2}$, ever more precise measurements are planned at Mainz. Great advances have also been made with the time-like form factors, but because of space constraints I will not discuss them, although the reader is referred to the contributions in parallel session A of this conference. In short, form factors are providing new insight into nucleon structure that are moving us toward a deep understanding of QCD in the non-perturbative regime.

\section{Formalism and measurement techniques}

The most general form for a relativistically covariant hadronic current for a spin-1/2 nucleon 
that satisfies current conservation is

$$
\mathscr{J}_{\text {hadronic }}^{\mu}=e \bar{N}\left(p^{\prime}\right)\left[\gamma^{\mu} F_{1}\left(Q^{2}\right)+\frac{i \sigma^{\mu v} q_{v}}{2 M} F_{2}\left(Q^{2}\right)\right] N(p),
$$

where $N(p)$ and $\bar{N}\left(p^{\prime}\right)$ are the nucleon Dirac spinors for the initial and final momenta $p$ and $p^{\prime}$, respectively, and $F_{1}\left(Q^{2}\right)$ and $F_{2}\left(Q^{2}\right)$ are the Dirac and Pauli form factors. It is often convenient to use the Sachs form factors that are linear combinations of $F_{1}$ and $F_{2}$ :

$$
G_{E}=F_{1}-\tau F_{2} \text { and } G_{M}=F_{1}+F_{2},
$$

where $\tau=\mathrm{Q}^{2} / 4 \mathrm{M}_{\mathrm{N}}^{2}$, and $\mathrm{M}_{\mathrm{N}}^{2}$ is the mass of the nucleon. In terms of the Sachs form factors, the differential cross section for elastic scattering can be written

$$
\frac{d \sigma}{d \Omega}=\left(\frac{d \sigma}{d \Omega}\right)_{\mathrm{Mott}}\left(G_{E}^{2}+\tau\left[1+2(1+\tau) \tan ^{2} \frac{\theta_{e}}{2}\right] G_{M}^{2}\right) /(1+\tau),
$$

where $\theta_{e}$ is the scattering angle in the lab frame and $\left(\frac{d \sigma}{d \Omega}\right)_{\text {Mott }}$ is the Mott cross section defined by $\sigma_{\text {Mott }}=\left(e^{2} / 2 E_{\text {beam }}\right)\left(\cos ^{2} \frac{\theta_{e}}{2} / \sin ^{4} \frac{\theta_{e}}{2}\right)$. Using Eq. 2.3, it is possible to disentangle the electric and magnetic form factors by performing measurements at a well-chosen set of values of both $Q^{2}$ and $\theta_{e}$, a process known as a Rosenbluth separation.

At high $Q^{2}$, there are at least two issues that represent significant challenges when performing Rosenbluth separations. One has long been recognized, that at higher values of $Q^{2}$ the term involving $G_{M}$ dominates, making it difficult to perform an accurate extraction of $G_{E}$. Another issue that has come to light in recent years, however, is more subtle. The validity of Eq. 2.3 depends on the one-photon or Born approximation. It has now been established that two-photon contributions to the cross section can, at high $Q^{2}$, be sufficiently large that the extraction of the form factors (using a Rosenbluth separation) is severely compromised.

The double-polarization method, which measures the ratio $G_{E} / G_{M}$, addresses the limitations of Rosenbluth separations at high $Q^{2}$. Two approaches are used: one involving a polarized beam and a recoil polarimeter, and the other involving a polarized beam and a polarized target. For the proton form factors, the best sensitivity has been achieved using the recoil polarimeter approach. In the one-photon exchange approximation the scattering of longitudinally polarized electrons from unpolarized hydrogen results in a transfer of polarization to the recoil proton with two components, $P_{t}$ perpendicular to, and $P_{\ell}$ parallel to the proton momentum in the scattering plane. Measuring these two components simultaneously and taking their ratio gives the ratio of the form factors:

$$
\frac{G_{E}}{G_{M}}=-\frac{P_{t}}{P_{\ell}} \frac{\left(E_{e}+E_{e}^{\prime}\right)}{2 m_{p}} \tan \frac{\theta_{e}}{2}
$$

where $E_{e}$ and $E_{e}^{\prime}$ are the initial and final electron energy. The neutron FF measurements have been performed using both techniques, involving either a polarized target or a recoil polarimeter. If we consider the situation in which the neutron polarization is both perpendicular to the momentum transfer and in the scattering plane of the electron, the double-polarization asymmetry can be written as

$$
A=\frac{-\left(G_{E} / G_{M}\right) \sqrt{4 \tau(1+\tau)} \tan \left(\theta_{e} / 2\right)}{\left(G_{E} / G_{M}\right)^{2}+\tau\left(1+2(1+\tau) \tan ^{2}\left(\theta_{e} / 2\right)\right)} .
$$


Whether working with a polarized target or a recoil polarimeter, the double-polarization asymmetry has only a negligible contribution from two-photon effects, and for high $Q^{2}$, it has become the gold standard for any form-factor measurement.

\section{The size of the nucleon}

Historically, elastic form factors played a central role in establishing the size of the nucleon. At low values of $Q^{2}$, when relativistic corrections are quite small, the electric and magnetic form factors are essentially the Fourier transforms of the distributions of charge and magnetization respectively. This picture breaks down at higher momentum transfers, although it is still possible to rigorously define transverse spatial distributions in the light-front frame that are essentially Fourier transforms of the Dirac and Pauli form factors in impact-parameter space [3, 4, 5]. These distributions, which should be distinguished from lab-frame densities, have some unusual properties. For example, as pointed out by Carlson and Vanderhaeghen, a transversely polarized neutron appears to have an "electric dipole moment" which is actually the boosted magnetic dipole [5]. Even so, these light-front distributions provide rigorous information on the nucleon's transverse structure.

At very low $Q^{2}$, the derivative of $G_{E}$ provides a measure of the charge radius. Recent very precise cross section data from Mainz as well as new polarization-transfer data from JLab provide significantly improved knowledge of the charge radius of the proton [6]. These new data from Mainz and JLab agree with one another as well as with charge-radius determinations extracted from Lamb Shift measurements on hydrogen. A new and very accurate measurement of the Lamb Shift in muonic hydrogen [7], however, is in sharp disagreement with these other determinations, a discrepancy that is as yet unresolved. Even more precise data from Mainz are planned.

\section{Measurements of $G_{E}^{p} / G_{M}^{p}$ and $G_{E}^{n} / G_{M}^{n}$}

Until roughly ten years ago, existing data indicated that the ratio of the electric and magnetic form factors of the proton, $G_{E}^{p} / G_{M}^{p}$, was relatively constant. Despite limits in the accuracy of these data at high $Q^{2}$, which were obtained almost exclusively using Rosenbluth separations, a bias evolved in which the ratio $G_{E}^{p} / G_{M}^{p}$ was expected to remain constant. This perception was changed abruptly by the double-polarization measurements of Jones et al. [1] who observed that above roughly $1 \mathrm{GeV}^{2}$, the ratio $G_{E}^{p} / G_{M}^{p}$ decreases almost linearly with increasing $Q^{2}$ (see Fig. 1). The discovery of this unexpected behavior prompted a significant reexamination of the nucleon's structure. It also raised the question of why measurements of $G_{E}^{p} / G_{M}^{p}$ relying on Rosenbluth separations disagreed so significantly with double-polarization measurements. The disagreement appears to be largely if not completely resolved when two-photon effects are included. It was soon apparent that most theoretical explanations of the $Q^{2}$ behavior of $G_{E}^{p} / G_{M}^{p}$ needed to invoke an important role for quark orbital angular momentum (OAM). It is quite notable that just as the importance of quark OAM was being established in understanding the $Q^{2}$ behavior of $G_{E}^{p} / G_{M}^{p}$, evidence of quark OAM began showing up in other unrelated experiments [8].

Among the theoretical explanations that explained well the behavior of $G_{E}^{p} / G_{M}^{p}$ were relativistic constituent quark models (RCQMs), that predicted the behavior before it was observed [9]. These models generally include a violation of hadron helicity conservation, and thus necessarily 


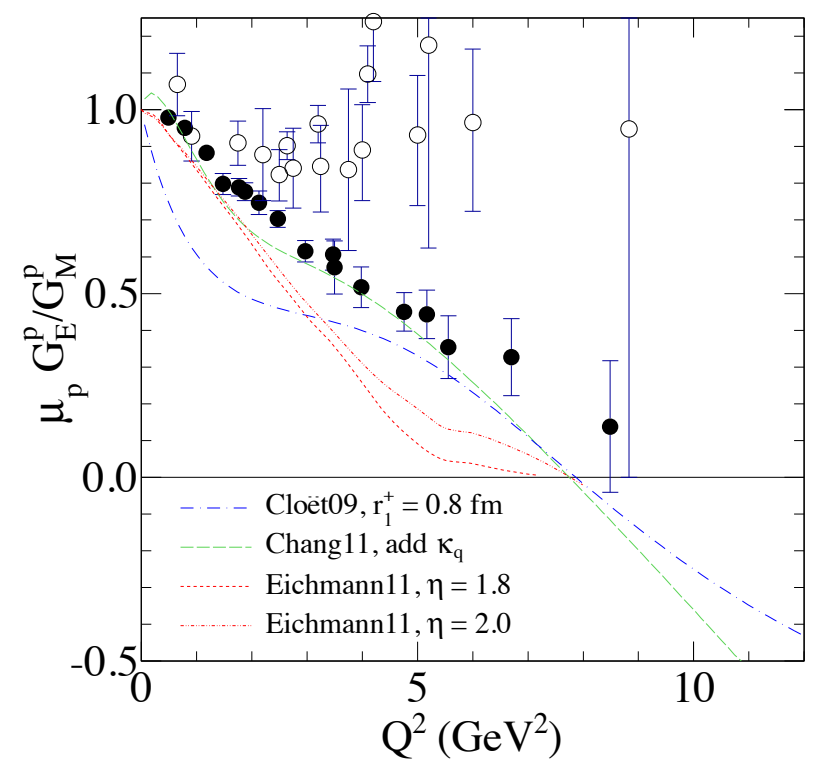

Figure 1: This figure, taken from Ref. 2, shows the final analysis of the three double-polarization measurements of $G_{E}^{p} / G_{M}^{p}$, the first of which was reported in Ref. 1. It also shows several measurements of $G_{E}^{p} / G_{M}^{p}$ obtained using Rosenbluth separations. The models shown are based on Dyson-Schwinger calculations.

include a significant role for quark OAM. Another example that represents a step toward an analytic approach are calculations based on QCD's Dyson Schwinger equations, in which the mass of the constituent quarks is dynamically generated [10]. The quarks are then coupled and the nucleon structure is computed using a Fadeev calculation. Again, quark OAM plays an important role, as do diquark degrees of freedom, a point that will be discussed more in the next section.

A subject that has gotten considerable attention is the scaling that one would expect for $G_{E}^{p} / G_{M}^{p}$ according to constituent counting rules. At sufficiently high values of $Q^{2}$, the Dirac form factor $F_{1}$ is expected to scale like $1 / Q^{4}$. Furthermore, the Pauli form factor $F_{2}$, because it involves a spin flip, is expected to have an additional factor of $1 / Q^{2}$. The quantity $Q^{2} F_{2}^{p} / F_{1}^{p}$ would thus be expected to approach a constant value at sufficiently high values of $Q^{2}$. Jones et al. noted that this did not occur over the $Q^{2}$ range they studied [1]. It was subsequently shown by Belitsky, Ji and Yuan that by relaxing hadron helicity conservation, and including components in the quark wave functions with orbital angular momentum $L>0$, the observed behavior could be reproduced by the resulting logarithmic corrections [11]. While one might interpret this as evidence for the importance of quark OAM, the authors were quick to point out that the values of $Q^{2}$ considered were sufficiently low that one might well be seeing "precocious scaling".

With high $Q^{2}$ measurements of the proton, there was considerable need to better understand the neutron in a similar $Q^{2}$ range. If nothing else, numerous theoretical calculations were published that described well the proton measurements, and while these calculations were understandably quite clustered for the existing proton data, they were quite divergent in their predictions for the neutron. Double-polarization measurements of $G_{E}^{n} / G_{M}^{n}$ using a recoil polarimeter had provided accurate data up to roughly $1.45 \mathrm{GeV}^{2}$, but obtaining reasonable statistics at any higher values of $Q^{2}$ appeared difficult. In 2010, Riordan et al. published measurements of $G_{E}^{n} / G_{M}^{n}$ from JLab 


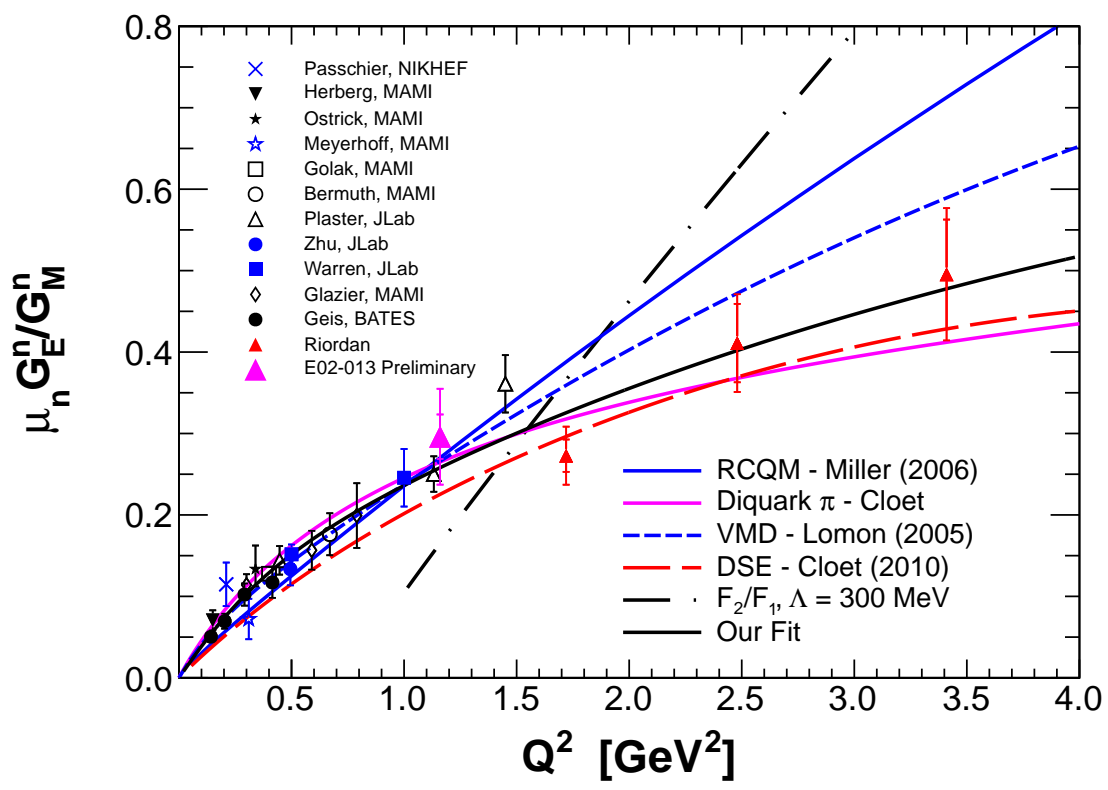

Figure 2: Data are shown for $G_{E}^{n} / G_{M}^{n}$, with the highest $Q^{2}$ values coming from Ref. 13 (E02-013), a doublepolarization experiment employing a polarized ${ }^{3} \mathrm{He}$ target. Included on this plot is the preliminary value from E02-013 for $Q^{2}=1.2 \mathrm{GeV}^{2}$.

E02-013 that extended up to $Q^{2}=3.41 \mathrm{GeV}^{2}$ [13]. The measurements were made possible by using a large-acceptance spectrometer, BigBite, based on a single dipole magnet, a high-luminosity polarized ${ }^{3} \mathrm{He}$ target, and what may have been the world's largest neutron detector. The results of those measurements, together with earlier measurements of $G_{E}^{n} / G_{M}^{n}$, are shown in Fig. 2. We note that Fig. 2 also shows a preliminary analysis of the lowest $Q^{2}$ point obtained by E02-013 that was not included in the initial Physical Review Letter of ref. [13].

The new measurements of $G_{E}^{n} / G_{M}^{n}$ from E02-013 were the first to probe well into the regime in which the unusual behavior was observed for $G_{E}^{p} / G_{M}^{p}$. The results, not surprisingly, fell well above the earlier predictions of the historical Galster parameterization, and generally agreed with the higher values predicted by theoretical efforts to understand the proton. It is worth noting that the prediction shown in Fig. 2 labeled DSE by Cloët et al. falls closest to the measured values.

\section{Flavor separated form factors}

If we assume charge symmetry it is possible to perform a flavor decomposition of the proton (neutron) form factors $F_{1}^{p(n)}$ and $F_{2}^{p(n)}$, and construct form factors corresponding to the individual matrix elements of the separate up and down quarks [14]. Here we use the relations

$$
F_{1(2)}^{u}=2 F_{1(2)}^{p}+F_{1(2)}^{n} \quad \text { and } \quad F_{1(2)}^{d}=2 F_{1(2)}^{n}+F_{1(2)}^{p} .
$$

In writing the above equations, we are using the standard convention in which $F_{1(2)}^{u}$ and $F_{1(2)}^{d}$ refer to the up and down quark contributions to the Dirac (Pauli) form factors of the proton.

As mentioned in the previous section, a quantity that was discussed in ref. [1] is $S_{p} \equiv Q^{2} F_{2}^{p} / F_{1}^{p}$, which is expected to become constant at sufficiently high $Q^{2}$. It is thus interesting to ask how cor- 
responding quantities for the up and down quarks behave. We thus define the quantities

$$
S_{u} \equiv Q^{2} F_{2}^{u} / F_{1}^{u} \quad \text { and } \quad S_{d} \equiv Q^{2} F_{2}^{d} / F_{1}^{d}
$$

and plot them in the bottom panel of Fig. 3a, where each individual data point corresponds to an experimental result on $G_{E}^{n} / G_{M}^{n}$ as is described in ref. [14]. We also show the quantity $S_{p}$ in the upper panel of Fig. 3a. What we see is quite striking. Whereas $S_{p}$, in the absence of logarithmic corrections, shows no signs of saturating, $S_{u}$ and $S_{d}$ for the individual quark flavors look at first glance quite linear! The explanation for this behavior lies in the fact that above roughly $1 \mathrm{GeV}^{2}$, the ratios $F_{2}^{u} / F_{1}^{u}$ and $F_{2}^{d} / F_{1}^{d}$ are remarkably constant. This is in violation of the usual expectation that $F_{2} / F_{1} \sim 1 / Q^{2}$, although it has been pointed out by Brodsky and Drell that this relation does not need to hold for a composite particle [15].
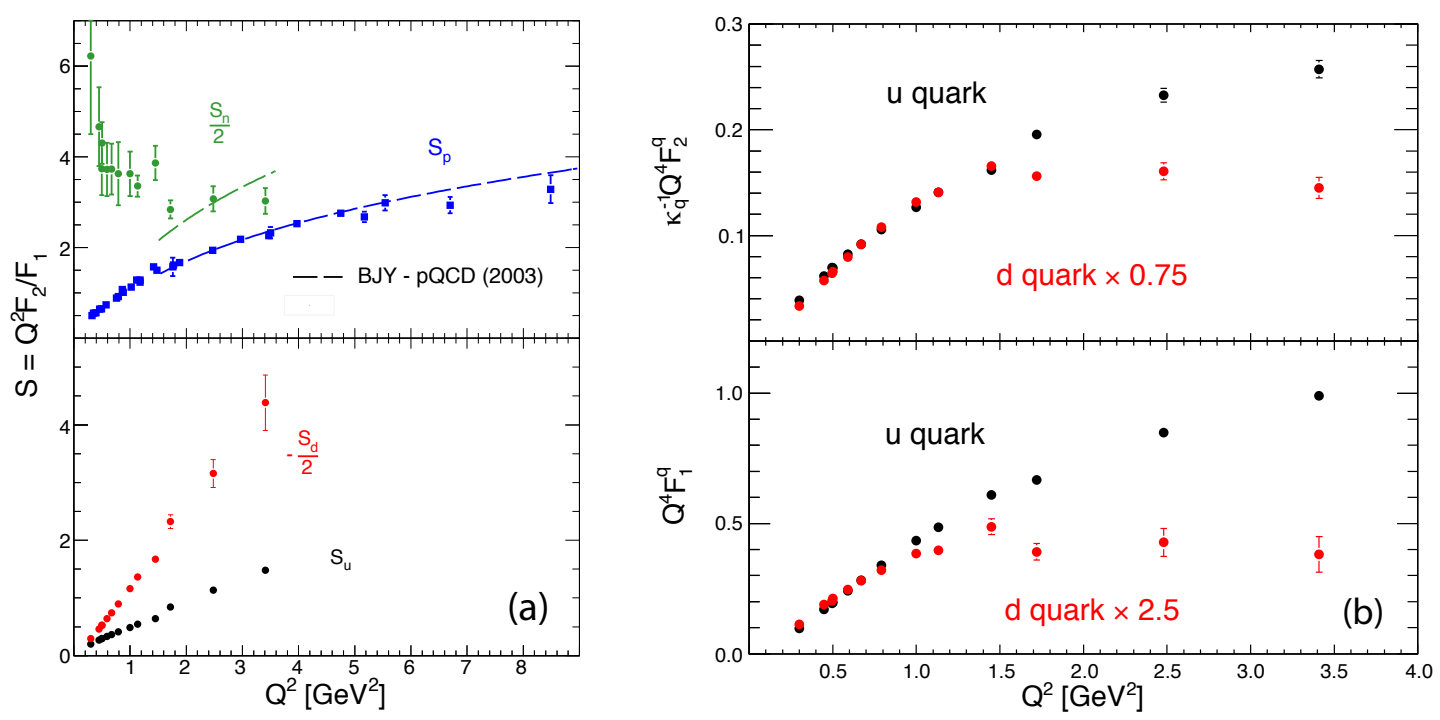

Figure 3: Shown are two figures from Ref. 14 illustrating the behavior of the flavor-separated form factors. See the text for details.

Another surprise with potentially important implications is illustrated in Fig. 3b, where $F_{2}^{u}$, $F_{2}^{d}, F_{1}^{u}$ and $F_{1}^{d}$ are all plotted, each multiplied by $Q^{4}$. For the down quark, both $F_{1}^{d}$ and $F_{2}^{d}$ appear to begin scaling like $1 / Q^{4}$ just above $1 \mathrm{GeV}^{2}$. In contrast, $F_{1}^{u}$ and $F_{2}^{u}$ are scaling more like $1 / Q^{2}$ if at all. Several theoretical examinations of this very different behavior for up and down quarks seem to point to the importance of diquark degrees of freedom.

A particularly important calculation in this context is the aforementioned approach that utilizes QCD's Dyson-Schwinger equations. In a recent paper by Cloët, Roberts and Wilson, the authors state in the abstract that they have computed " ... a prediction for the $Q^{2}$-dependence of $u$ - and $d$ quark Dirac and Pauli form factors in the proton, which exposes the critical role played by diquark correlations with the nucleon." [16] Another interesting calculation by Cloët and Miller based on the RQCM also reproduces the $Q^{2}$ behavior of the flavor-separated form factors by including diquark terms in their wave functions. This approach also reproduces the fraction of the nucleon's spin carried by the spins of the quarks as measured in deep inelastic scattering. 
Finally, we mention an interesting suggestion by Miller that has yet to be explored formally [17]. We imagine that the nucleon is dominated by an isoscalar diquark containing a $u$ - and a $d$-quark, and a single lone $u$ quark. If elastic scattering off a $u$ quark were dominated by the lone $u$ quark, the nucleon would look like an object with two constituents, and $F_{1}^{u}$ would scale like $1 / Q^{2}$. Scattering off a $d$ quark would then necessarily involve the diquark, which to hold together during elastic scattering, would require the exchange of a second hard gluon. The Dirac form factor $F_{1}^{d}$ would then be expected to scale like $1 / Q^{4}$. If correct, this would provide a wonderfully simple explanation for the behavior. For now, however, it should be viewed strictly as an idea worth further consideration.

\section{Conclusions}

If we go back roughly a decade, a common picture of the nucleon involved three separate quarks in which quark OAM did not play a significant role. The possibility of a diquark was taken seriously because of how neatly it would explain missing states in the $N^{*}$ spectrum, but the "cartoons" we draw of the nucleon to this day do not contain such a structure. Now, however, quark OAM is taken quite seriously, and flavor-separated form factors seem to point to diquarklike structures. Regardless of how these issues are resolved, it is clear that the elastic form factors are continuing to provide information that is central to our understanding of nucleon structure.

\section{References}

[1] M. K. Jones et al. [Jefferson Lab Hall A Collaboration], Phys. Rev. Lett. 84, 1398 (2000).

[2] A.J.R. Puckett et al. (Jefferson Lab Hall A Collaboration), Phys. Rev. C 85, 045203 (2012).

[3] M. Burkardt, Phys. Rev. D 62, 071503 (2000); Int. J. Mod. Phys. A 18, 173 (2003);

[4] G. A. Miller, Phys. Rev. Lett. 99, 112001 (2007);

[5] C. E. Carlson and M. Vanderhaeghen, Phys. Rev. Lett. 100, 032004 (2008).

[6] J.C. Bernauer et al., Phys. Rev. Lett. 105, 242001 (2010); X. Zhan et al.,Phys. Lett. B 705, 59 (2011).

[7] B. Pohl, et al., Nature 466 (2010) 213.

[8] X. Zheng et al., Phys. Rev. Lett. 92, 012004 (2004); A. Airapetian et al., Phys. Rev. Lett. 103, 152002 (2009).

[9] See for example the review article: C.F. Perdrisat, V. Punjabi and M. Vanderhaeghen, Progress in Particle and Nuclear Physics 59, 694Đ764 (2007).

[10] I. C. Cloët et al., Few-Body Syst. 46, 1 (2009).

[11] A.V. Belitsky, X. D. Ji, and F. Yuan, Phys. Rev. Lett. 91, 092003 (2003).

[12] Ian C. Cloët and Gerald A. Miller, arXiv:1204.4422v2 [nucl-th] (2012).

[13] S. Riordan et al., Phys. Rev. Lett. 105, 262302 (2010).

[14] G.D. Cates, C.W. de Jager, S. Riordan and B. Wojtsekhowski, Phys. Rev. Lett. 106, 252003 (2011).

[15] Stanley J. Brodsky and Sidney D. Drell, Phys. Rev. D 22, 2236 (1980).

[16] I.C. Cloët, C.D. Roberts and D. J. Wilson, arXiv:1103.2432v1 [nucl-th] (2011).

[17] Gerald Miller, private communication. 